\section{Allergy Immunology}

\title{
Associate Editor Motohiro Ebisawa
}

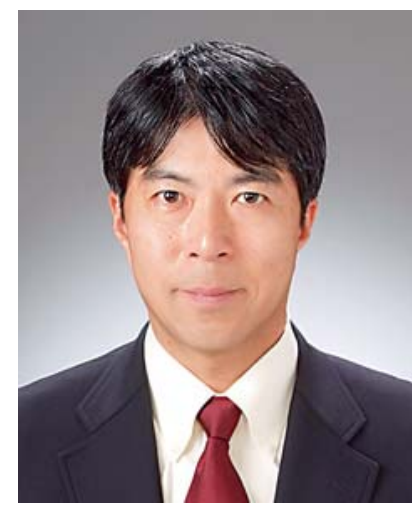

Motohiro Ebisawa, Kanagawa.
Motohiro Ebisawa is the Director of Allergy at the Clinical Research Center for Allergy and Rheumatology, Sagamihara National Hospital, and the Visiting Professor of The Jikei University School of Medicine. He graduated from The Jikei University School of Medicine in Tokyo, Japan, in 1985 and took a short, visiting residence course at the Department of Pediatrics at St. Thomas Hospital in London, UK. He completed his postgraduate studies at The Jikei University School of Medicine, where he earned his PhD. He worked on a postdoctoral fellowship on the analysis of "mechanisms of eosinophilspecific recruitment" in Dr. Robert P. Schleimer's laboratory at Johns Hopkins University from 1991 to 1993 . After returning to Japan, he commenced food allergy practice and research at Sagamihara National Hospital, which has been his focus since 1995. He has won multiple awards, including the Allergy 2000 International Young Investigator Award, and has authored more than 100 articles in peer-reviewed international publications.

He is a leading expert in food allergies and currently serves as the Secretary General on the Board of Directors of WAO, where he has served in different leadership roles since 2010 (Member at Large, 2010-2013, and Treasurer, 2014-2015). In addition to his WAO tenure, he serves on the Executive Board of Directors of the Japanese Society of Allergology (JSA), the Board of Directors of the Japanese Society of Pediatric Allergy and Clinical Immunology (JSPACI), and the Board of Directors of the Asia Pacific Association of Pediatric Allergy, Respirology and Immunology (APAPARI). He is a Chair of the Food Allergy Committee of JSPACI and a member of the Collegium Internationale Allergogica (CIA). He also plays a role as an Associate Editor of International Archives of Allergy and Immunology and is a member of the editorial boards of The Journal of Allergy and Clinical Immunology: In Practice, Pediatric Allergy and Immunology, and Allergy, Asthma \& Clinical Immunology, as well as a member of review boards of The Journal of Allergy and Clinical Immunology. 